\title{
Graphic data sharing
}

Daniel Tena-Parera, Universitat Autònoma

de Barcelona, Daniel.tena@uab.cat

RESUMEN.

Debemos reflexionar en el futuro inmediato sobre los datos de las investigaciones científicas y su uso. El texto expone la tendencia a la compartición de datos que determinados ámbitos científicos ya establecen como requisitos para la publicación de artículos. En el futuro inmediato grafica pretende fomentar esta tendencia.

\section{Palabras clave}

Diseño gráfico; data sharing; investigación

\section{Graphic data sharing}

\section{ABstRact}

We must reflect in the immediate future on the data of scientific research and its use. The text exposes the tendency to share data (data sharing) for the publication of articles. In grafica, we intend to encourage this trend.

\section{Keywords}

Graphic design; neologisms; significance
Nunca antes había iniciado un texto con tanto interés como éste y con una trascendencia, quizás mayor que la que pueda tener éste. Quizás lo que aquí se expondrá, no va a suponer ningún elogio, ningún cambio o ningún reproche. Sea como sea, lo mas interesante para quien escribe es que se nos presenta por delante un contenido de futuro que bien debería ser el presente.

Nuestro interés no es tanto que se quiera, pueda o deba exponer muchas cosas. Todo lo contrario, la idea es bien simple, concreta y espero que clara.

¿Cual es el bien más preciado de la investigación? ¿Son los indicios de nuevo conocimiento? ¿Son los datos obtenidos? ¿Es la capacidad del investigador 0

investigadora para reproducir un proceso científico? ¿Es la transmisión de ese pretendido nuevo conocimiento? Todas estas preguntas y algunas más que nos podamos formular tienen quizás múltiples respuestas. Es decir, el grado de opciones de las respuestas puede ser tendente al infinito. No hay duda que han sido formuladas con carácter retórico y con la pretensión de contextualizar el problema y la solución que aquí se pretende presentar.

Efectivamente, pretendemos poner el foco de atención en un factor al que se presta poca importancia atención.

\section{Los datos de la investigación}

Sin duda cuando se aborda una investigación, el tema principal al que se enfrenta el investigador o investigadora es formular buenas hipótesis o, en su defecto, adecuadas preguntas de investigación. Esta cuestión solo requiere tiempo y clarividencia para formular una especulación, en forma de hipótesis, que nos lleve a un conocimiento no disponible todavía. El uso de un tamaño en un objeto y su posición en el espacio gráfico, la cantidad de elementos, su textura, forma, color o cualquier otra variable independiente que se nos pueda vislumbrar en un objeto de estudio con- 
creto requerirán de nuestra argucia intelectual i visión de futuro: tiempo, visión y quizás una cierta experiencia serán usadas para ello.

A partir de ese momento aparece un segundo y más complejo problema. ¿Cómo vamos a corroborar, validar, estudiar... las variables que nuestro artilugio especulativo haya designado? Es decir, ¿Con qué datos?

Por lo general, los datos los obtenemos del estudio de campo. La investigación a partir de entrevistas en profundidad, la investigación participante, e incluso el análisis de contenido pueden ser el origen de unos datos que posteriormente, y debidamente analizados, nos reporten certezas acerca de nuestras hipótesis. También los test de percepción, de usabilidad, de accesibilidad, de comprensión... pueden ser fuente de obtención de datos que van a permitirnos la validación científica. Incluso, acceder al banco de datos estadísticos nos pueden permitir determinas aseveraciones científicas.

Sea cual sea el origen de los datos, sin ellos no podemos investigar. Además, generalmente, su obtención es costosa en tiempo y, en cualquiera de los casos, un derroche en recursos económicos que no todas las investigaciones disponen.

Efectivamente, los datos son aquel bien preciado que toda investigación requiere. Su credibilidad, validez y calidad se les supone de antemano. Pero su longevidad es, a menudo, excesivamente escasa. No por su vigencia, más bien por la capacidad que tenemos de acceder a ellos y encontrarles múltiples usos, más allá de la investigación para los que han sido obtenidos.

\section{Socializando datos}

De esta limitada visión y utilización de los datos aparece una tendencia a su socialización. Si bien no es una tendencia nueva en determinados ámbitos de la ciencia, en otros es una cuestión que nos puede coger por sorpresa.

Nos referimos al "Data Sharing" o tendencia a compartir datos de investigación en base a su denominación anglosajona (Fienberg et al: 1985). El Data Sharing consiste en la actividad de facilitar los datos de una investigación para que puedan ser utilizados por otros investigadores. Es decir, se trata de compartir e intercambiar los metadatos que toda investigación posee.

"The Royal Society" establece, para cualquier revista científica, que la compartición de datos forme parte de la cultura de la investigación científica. Indica que es una condición para las revistas bajo su institución que los autores de los artículos pongan a disposición de otros investigadores los datos, el código y los materiales de investigación que respaldan los resultados del artículo" (https://royalsociety.org/journals/ethicspolicies/data-sharing-mining/; Fecha de consulta: junio 2019).

No se trata de un fenómeno aislado, el data sharing es una tendencia al alza. Ya se da por hecho que el data sharing es una ventaja no solo para el autor, productor o propietario de los datos. Lo es también para el investigar o investigadora que los puede reutilizar. Mientras muchas veces los datos quedan ahí, escondidos, olvidados; con el data sharing recuperan todo el protagonismo que les corresponde.

Todos salen ganando con la compartición de los datos de una investigación. Autores, otros investigadores, la ciencia en general y, naturalmente, la sociedad. Se rentabilizan los esfuerzos de investigación al amortizar mejor los datos que una investigación obtiene.

\section{Data sharing y las revistas científicas}

Esta tendencia no será opaca para las revistas científicas y, cada vez más, éstas solicitaran a los autores de un artículo científico que va a ser revisado por pares la información complementaria y necesaria para comprender, desarrollar o reproducir la investigación que se desea publicar. Esto es: datos sin procesar, métodos estadísticos o código fuente. Es decir, lo que podemos denominar los metadatos de los datos de la investigación.

Actualmente las revistas que solicitan la compartición no lo requieren como requisito obligatorio. La compartición se establece más como una voluntad de los autores e investigadores que como un requisito para publicar un artículo.

No todas las cuestiones son tan positivamente evidentes. Existen por el momento, ciertas limitaciones de carácter legal para la compartición de datos: protección de datos, intereses de propiedad, la confidencialidad o el uso inadecuado de los datos.

En cualquier caso, no dudamos que los datos y su uso es, y será, un campo de gran interés por su condición de factor estratégico para el desarrollo e innovación las revistas científicas. Y lo serán también para las instituciones que subvencionan las investigaciones. Éstas ya empiezan a establecer políticas orientadas a los autores e investigadores para que compartan los datos de sus investigaciones. Todo ello va a estar favorecido por el desarrollo de las tecnologías de la información y del uso de ella en acceso abierto. 


\section{Datos en bruto}

Vamos a continuación a concretar lo que debemos considerar como los metadatos de los datos; es decir, los datos en bruto.

Para una primera apreciación de los datos en bruto puede servirnos el símil para indicar que estamos hablando de las fuentes documentales de las investigaciones.

En este sentido tenemos que considerar como datos en bruto a las fuentes documentales primarias y las secundarias. Por datos o fuentes primarias debemos considerar todas y cada una de las informaciones que los investigadores pueden obtener directamente de la fuente. Es decir, el estudio de campo, las entrevistas, la observación directa son primarias en la medida que el investigador entra en contacto con los datos en su fase de obtención. En definitiva, todo aquel dato obtenido directamente de la fuente.

Por otro lado, debemos hablar de los datos o fuentes secundarias como aquellas que han estado elaboradas por terceras personas a partir de otras fuentes secundarias o también, a partir de fuentes primarias. En este grupo encontramos informes y ordenación de datos... En definitiva, cualquier documento publicado o no que nos permita, a partir de él, realizar una investigación. La información en forma de resultados estadísticos son un claro ejemplo de este tipo de datos. Pueden proceder de instituciones públicas o privadas.

En este sentido, una de las cuestiones relevantes sobre la compartición de datos es determinar qué son y qué no son datos. No son datos de investigación y, por tanto, no susceptibles de ser compartidos los borradores para la elaboración de un artículo, ni los análisis preliminares, ni los proyectos de una investigación, ni las revisiones por pares, ni ninguna otra información entre miembros de un grupo de investigación. Sí lo son los datos estadísticos, los datos tabulados, los contenidos "en bruto" a partir de los cuales realizamos una investigación, las trascripciones de entrevistas, la catalogación de documentos, etc.

Un buen ejemplo y cercano de esta idea de compartición de datos lo podemos encontrar en el artículo publicado en "Questiones Publicitarias" \# 18, p 144-159 (Tena: 2013). El artículo bajo el título "El fondo de recursos digitales para la docencia e investigación en los estudios de Publicidad y Relaciones Públicas" es la presentación de 7.000 anuncios gráficos publicitarios. Se expone que están indexados según criterios académicos y profesionales: sector, categoría, producto, anunciante y marca, texto, año, forma- to, color, imagen, suporte, tipografía, estética, los actores, las acciones, etc. Y lo que es más importante, se ponen a disposición en abierto (open acces) para aquellos investigadores que deseen acceder a ellos para sus investigaciones. Creemos que es un buen punto de partida. $Y$ no en vano, a partir del uso de ese fondo se publicó el año pasado en "grafica" el artículo científico "Comunicando a través de la seducción: análisis de la dirección de arte en la publicidad de perfumes" (Balbuena \& López) en grafica vol. 7, No 13, p. 53-62.

Los datos pueden ser de carácter cuantitativo y cualitativo. Entre los primeros encontramos los datos obtenidos de cuestionarios estadísticas que pueden ser de organizaciones públicas o privadas. Así el Instituto Nacional de Estadística (INE) o el Centro e Investigaciones Sociológicas (CIS) ofrecen un gran volumen de información en bruto. Del mismo modo, podemos encontrar datos vinculados a entrevistas, focus group o test de percepción, entre otros sistemas de recogida de información que pueden ser considerados como datos en bruto.

\section{Líneas futuras}

¿Vamos a compartir nuestros datos o vamos a guardárnoslos? ¿Vamos a ofrecer de manera gratuita los datos en bruto que tanto nos ha constado obtener? O por el contrario no vamos a dejar que nadie pueda acceder a ellos? Ciertamente, podemos detectar ciertos frenos: ¿por qué facilitar aquello que nos ha costado tanto obtener? Simplemente, hacerlo nos va a favorecer.

Vamos a tener más visibilidad. Nuestros datos nos van a generar mayor indexación.

La compartición de nuestros datos nos permite mayor disponibilidad para el estudio, análisis y establecer buenas estrategias de investigación; $y$, finalmente, mayor tiempo para la transferencia (figura 1). A veces seremos nosotros los autores, pero a veces seremos nosotros los receptores de los datos de otros.

Si las amenazas de la compartición de datos son menores que las ventajas pasaremos a una segunda etapa donde los problemas serán ya de carácter técnico:

1. Mayores y mejores infraestructuras para la cantidad de datos a compartir.

2. Establecer los protocolos comunes de acceso a los datos.

Los planes futuros y la perspectiva en grafica respecto del Data Sharing podríamos convenirla en la progresiva apuesta por el data sharing. Para 


\section{DRYAD} About For researchers For organizations $\mathrm{C}$

Data from: A scenario for the evolution of selective egg colouration: the roles of enemy-free space, camouflage, thermoregulation, and pigment limitation

Torres-Campos I, Abram PK, Guerra-Grenier E, Boivin G, Brodeur J Date Published: March 9, 2016

DOI: https://doi.org/10.5061/dryad.5qt2k

\section{Files in this package}

Content in the Dryad Digital Repository is offered "as is." By downloading files, you agree to the Dryad Terms of Service. To the extent possible under law, the authors have waived all copyright and related or neighboring rights to this data. (Ge) ZEFO

\begin{tabular}{|c|c|}
\hline Title & Field and Laboratory tests data_IT-C \& PKA et al. \\
\hline Downloaded & 179 times \\
\hline Description & $\begin{array}{l}\text { Field tests data: development time, survival no tanglefoot and survival } \\
\text { tanglefoot; Laboratory tests data: starvation per eggmass, starvation per } \\
\text { females and temperature eggmass. }\end{array}$ \\
\hline Download & Field and Laboratory tests data IT-C \& PK...I.zip $(12.01 \mathrm{~Kb})$. \\
\hline Details & View File Details \\
\hline
\end{tabular}

When using this data, please cite the original publication:

Torres-Campos I, Abram PK, Guerra-Grenier E, Boivin G, Brodeur J (2016) A scenario for the evolution of selective egg colouration: the roles of enemy-free space, camouflage, https://doi.org/10.1098/rsos.150711

Additionally, please cite the Dryad data package:

Torres-Campos I, Abram PK, Guerra-Grenier E, Boivin G, Brodeur J (2016) Data from: A scenario for the evolution of selective egg colouration: the roles of enemy-free space, camouflage, thermoregulation, and pigment limitation. Dryad Digital Repository. https://doi.org/10.5061/dryad.5qt2k

Cite I Share

Figura 1. El repositorio digital de DYRAD es un recurso curado que hace que los datos subyacentes a las publicaciones científicas se puedan descubrir, reutilizar y citar libremente.

ello, la comunidad científica del entorno de grafica debe orientarse hacia:

1. La compartición bajo la petición al autor.

2. Facilitar el material como complemento a los artículos.

3. Trabajar para colocar los contenidos en un repositorio Open Acces.

Los modelos a seguir son los modelos multidisciplinares (Zenodo, Dyrad, Dataverse, Figshare); y los modelos propios de las ciencias sociales (Inter-university Consortium for Political and Social Research, UK Data, The Qualitative Data Repository; o bien plataformas institucionales (Sixto et alt: 2019).

Por el momento, las ciencias y las biomedicinas van a seguir siendo las más proclives a la compartición de datos mientras que las artes, humanidades y ciencias sociales van a seguir sus pasos.

\section{Grafica V7 \#14}

En el presente número de grafica publicamos tres artículos de investigación y cuatro reseñas. Para ello, hemos seguido el proceso de revisión ciega de doble pares que sigue "grafica" para la evaluación de los originales.

Adicionalmente, este número de grafica cuenta con un artículo en la sección "Expertia" sobre los 100 años de la Bauhaus. Joan Costa, autodidacta, comunicólogo, sociólogo, diseñador, metodólogo y referente para muchos diseñadores nos presenta una trayectoria histórica de la Bauhaus a través de ciertos "hitos" que la Escuela alemana ha logrado durante su historia.

"La estética gráfica de un libro impreso. Una exploración en la temática de ficción narrativa" es un artículo científico en el que Rafael Pozo nos describe el nuevo término "estética gráfica", como el resultado final que percibe un receptor cuando sostiene y observa un libro impreso entre sus manos. El trabajo plantea una teoría sobre la estética gráfica de un libro impreso y muestra evidencias que permiten aportar una primera evaluación desde las perspectivas cualitativa, educativa y científica.

El segundo artículo científico que encontraremos es de Beatriz Martínez Villagrasa "Creative Decoding Tool: una herramienta para la medición de las competencias de los diseñadores". En investigaciones previas, se desarrolló la Creative Decoding Tool (CDT), una encuesta online que mide una serie de competencias centrales en los procesos creativos de los diseñadores y que resulta útil como herramienta para fomentar la reflexión personal. En el presente artículo se analiza la primera muestra recogida, compuesta por 599 individuos. Los resultados nos orientan a la constatación de la existencia de diferencias significativas en las competencias profesionales en función de características sociodemográficas.

Por lo que hace referencia a los artículos científicos, un tercer y último "paper" nos permite acercarnos al diseño de juegos de mesa y el uso de los códigos de diseño que ello implica. Se trata del artículo "Adaptación y equivalencia de códigos en el diseño de un juego de mesa para discapacitados visuales: código gráfico y háptico" de Judit Vargas. El texto pone sobre la mesa aspectos de accesibilidad y la intervención de otros sentidos cuando no está presente el de la visión. En este sentido, se aborda el diseño inclusivo. El método se inició con la definición de las variables independientes (textura y tamaño) y la dependiente (identificación de elementos), se construyeron los prototipos de un juego concre- 
to y se verificó la propuesta mediante un test de recepción. Los resultados permiten establecer la equivalencia de códigos para que los juegos puedan ser usados tanto por personas con discapacidad visual como por las que no la tienen.

También en este número de grafica encontraremos una reseña de Rafael Pozo sobre "Signos de corrección de imprenta. Norma UNE 54151". Se trata de un toque de atención y dar relevancia a los signos de corrección que sintetizan códigos de órdenes diseñadas para facilitar la comunicación entre los profesionales de la edición y la impresión. Es decir, la comunicación técnica entre los diferentes agentes participantes en los procesos de la edición.

Otra reseña de las que se presentan en este número de grafica hacemos mención de la Exposición "El boom de la Publicidad" que el Museo de Diseño de Barcelona ha realizado des de diciem- bre de 2018 hasta el primer trimestre de 2019. En este sentido, se hace mención de las vinculaciones entre el diseño gráfico y la publicidad.

También la reseña de Manel Vinyals, toma como origen el Museo de Diseño de Barcelona. En este caso, para hablar de "Jornada de creatividad y tendencias. Museo del Diseño". La IV Jornada de creatividad y tendencias, organizada por el Museo del Diseño de Barcelona se focalizó en el diseño de experiencias.

Finalmente, podremos leer una reseña de Joan Costa sobre el libro conjunto de Pla-narbona y Joan Costa "Axiomagrafies", libro que conecta el pensamiento con la imagen.

Solo nos queda agradecer su colaboración a todos cuantos hacen posible este proyecto y esperar que este número sea del interés de los lectores.

Buena y productiva lectura. 


\section{Referencias bibliográficas}

Balbuena, L. \& López, Jesús, (2019) "Comunicando a través de la seducción: Análisis de la dirección de arte en la publicidad de perfumes". grafica Vol. 7, Núm. 13, . DOI: https:// doi.org/10.5565/rev/grafica.133

DYRAD (2019) https://datadryad.org/resource/ doi:10.5061/dryad.5qt2k

DRYAD (2019) Imatge: https://datadryad.org/resource/doi:10.5061/dryad.5qt2k

Fienberg, S. E., Martin, M. E., \& Straf, M. L. (Eds.). (1985). "Sharing research data". Washington, DC, US: National Academy Press.
Sixto Andrea, Aleixandre Rafael, Alonso Adolfo, Lucas Rut, y Vidal Antonio. (2019). "Hacia un modelo de evaluación de las políticas editoriales sobre uso compartido de datos de investigación en revistas de Ciencias Sociales". CRECS 2019. Logroño. España

Tena-Parera, D. Fondo documental Digital de Anuncios Gráficos

Publicitarios, (2013) Questiones publicitarias, Vol. I, No 18, https://doi.org/10.5565/rev/qp.44

The Royal Society. Data Sharing and mining (2019), https://royalsociety.org/journals/ ethics-policies/data-sharing-mining/ 